\title{
Millimeter-Wave Response of All Metal-Organic Deposited YBCO Transition Edge Bolometer
}

Nazifi, Rana; Mohajeri, Roya; Alipour, Zeynab; Mirzaei, Iman; Ahmadi-Boroujeni, Mehdi; Vesaghi, M. A.; Grivel, Jean-Claude; Fardmanesh, Mehdi

Published in:

IEEE Transactions on Applied Superconductivity

Link to article, DOI:

10.1109/TASC.2020.3033413

Publication date:

2021

Document Version

Peer reviewed version

Link back to DTU Orbit

Citation (APA):

Nazifi, R., Mohajeri, R., Alipour, Z., Mirzaei, I., Ahmadi-Boroujeni, M., Vesaghi, M. A., Grivel, J-C., \& Fardmanesh, M. (2021). Millimeter-Wave Response of All Metal-Organic Deposited YBCO Transition Edge Bolometer. IEEE Transactions on Applied Superconductivity, 31(1), [2100105]. https://doi.org/10.1109/TASC.2020.3033413

\section{General rights}

Copyright and moral rights for the publications made accessible in the public portal are retained by the authors and/or other copyright owners and it is a condition of accessing publications that users recognise and abide by the legal requirements associated with these rights.

- Users may download and print one copy of any publication from the public portal for the purpose of private study or research.

- You may not further distribute the material or use it for any profit-making activity or commercial gain

- You may freely distribute the URL identifying the publication in the public portal 


\title{
Millimeter-Wave Response of All Metal-Organic Deposited YBCO Transition Edge Bolometer
}

\author{
Rana Nazifi, Roya Mohajeri, Zeynab Alipour, S. Iman Mirzaei, Mehdi Ahmadi-Boroujeni, M.A.Vesaghi, \\ J.-C. Grivel, Mehdi Fardmanesh, Senior Member, IEEE
}

\begin{abstract}
We report on the response of a monolithic high $\mathbf{T}_{\mathrm{c}}$ transition-edge bolometer to about 3-mm-wave for the first time. The detector structure consisting of $400 \mathrm{~nm} \mathrm{YBa} \mathrm{Yu}_{3} \mathrm{O}_{7-\mathrm{x}}$ (YBCO) film on buffered Yttria Stabilized Zirconia substrate without any coupled antenna, shows bolometric type responsivity to the 3-mm-waves radiation at its transition temperature. The YBCO thin film and $\mathrm{Ce}_{0.9} \mathrm{La}_{0.1} \mathrm{O}_{2}$ buffer layer are both fabricated by the metal-organic deposition method. The meander line pattern of the bolometer is designed for obtaining maximum absorption and responsivity possible when the polarized radiation of the source is aligned with the pattern. Meander lines are 50 micrometers wide and $1.5 \mathrm{~mm}$ long. We have measured amplitude and phase of the response versus modulation frequency of the detector to the linearly polarized $95 \mathrm{GHz}$ source, and the detector was biased at 5 distinct temperatures at the transition corresponding to five different electrical conductivities of the YBCO film. When the meander lines of the device are parallel to the incident beam polarization, the YBCO pattern is speculated to act as a dissipative antenna resulting in higher absorption leading to high magnitude of the response as observed. The results from the measured phase of the response versus modulation frequency are also in agreement with the discussed absorbed mechanism. The absorption of the YBCO pattern is also measured to depend on the electrical conductivity of the YBCO film and our results show that there is an optimum electrical conductivity for having maximum absorption for this detector. Simulation results for this structure confirm the experiments showing that at electrical conductivity value of $1.33 \times 10^{5} \mathrm{~S} / \mathrm{m}$ we have the maximum absorption for our device. These observations promise design of versatile $\mathrm{THz}$ and millimeter-wave detectors with potentials for applications in medical and security imaging.
\end{abstract}

Index Terms-Bolometer, superconducting devices, YBCO, millimeter wave, terahertz ( $\mathrm{THz})$, detector.

\section{INTRODUCTION}

$\mathbf{S}$ UPERCONDUCTING transition-edge bolometer (TEB) are known for high detectivity, high speed, and no limitation in the wavelength of radiation they can detect. Since the discovery of superconductors, many groups have been working on increasing the sensitivity and wavelength detection range of the low $\mathrm{T}_{\mathrm{c}}$ and high $\mathrm{T}_{\mathrm{c}}$ superconducting bolometers [1]-[4]. From the wide wavelength range of these detectors, $\mathrm{THz}$ range with many applications from bioimaging to cosmic microwave

R. Nazifi and M.A.Vesaghi are with the Department of Physics, Sharif University of Technology, Tehran 11155-9161, Iran

R. Mohajeri, Z. Alipour, S. Iman Mirzaei, M. Ahmadi-Boroujeni, and M. Fardmanesh are with Electrical Engineering Department, Sharif University of Technology, Tehran 11155-9161, Iran (e-mail:fardmanesh@sharif.edu)

J.-C. Grivel is with Department of Energy Conversion and Storage, Technical University of Denmark, 2800 Kgs Lyngby, Denmark

S. Iman Mirzaei is presently with Department of Physics, Faculty of Basic Sciences, Tarbiat Modares University, P.O. Box 14115-175, Tehran, Iran background radiation detection, have attracted much attention to the superconducting bolometers [4]-[6].

The low reflectivity of the high $\mathrm{T}_{\mathrm{c}}$ superconductor $\mathrm{YBa}_{2} \mathrm{Cu}_{3} \mathrm{O}_{7-\mathrm{x}}(\mathrm{YBCO})$ at optical and near-infrared range makes them a good monolithic broadband detector for this range [7], [8]. At longer wavelengths when the photon energy becomes smaller than the superconducting gap, YBCO becomes mainly reflective [9], [10], and an antenna or absorbing layer is necessary to couple the radiation power to the YBCO detecting element. For millimeter and sub-millimeter wave detection, many groups have worked on antenna-coupled micro and nano bolometers, mostly with low $\mathrm{T}_{\mathrm{c}}$ superconductors [11], [12]. Recently, Kuzmin et al. reported an antennacoupled, voltage biased transition edge sensor for 1-6 THz [13], where the superconducting material is $5 \mathrm{~nm}$-thin $\mathrm{NbN}$, and the operating temperature of the device is $4.2 \mathrm{~K}$. Also, Hammer et al. [14] reported a $\mathrm{THz}$ detector based on spiral antenna coupled YBCO microbolometer with responsivity of $190 \mathrm{~V} / \mathrm{W}$ across the frequency range of $330 \mathrm{GHz}$ to $1.63 \mathrm{THz}$. Log-spiral [15], [16], slot antenna [11], and sinuous antenna [17] are different types of antennas which are mostly used in this era. Although, antenna-coupled structures show very high sensitivity, they have many steps in the fabrication process that make them complicated and expensive to fabricate.

In the present work, we propose a new bolometer design for the millimeter-wave detection, fabricated entirely by metalorganic deposition (MOD) growth technique. This device benefits coupling with the incident radiation due to its meander line pattern without any coupled antenna. This new design is promising for low cost fabrication of large area single pixel and arrays of this type of detectors. These economical detectors that work at temperatures above the liquid nitrogen temperature are good candidates for medical and security imaging applications. Here, we report the measured response of a designed YBCO TEB biased at its transition temperature of about $88 \mathrm{~K}$ illuminated by $95 \mathrm{GHz}$ radiation. The effect of YBCO thin film electrical conductivity on the detector response is also investigated.

\section{FABRICATION AND MEASUREMENT}

The device is made of $400 \mathrm{~nm}$ thick epitaxial YBCO layer grown on crystalline Yttria Stabilized Zirconia (YSZ) substrate by MOD method explained elsewhere [18]. We chose the YSZ substrate since it has the lowest thermal conductivity among the substrates for YBCO films leading to higher responsivity [18]. We have made some nano-scale roughness on the surface 
of the substrate with diamond nano-particles in order to obtain higher thermal boundary resistance between the film and the substrate as well as obtaining higher resistivity for the YBCO film. AFM microscopy shows the root-mean-square of the surface roughness equal to $2.7 \mathrm{~nm}$. The voltage response of the device is increased compared to the non-treated substrate, due to an increase of the electrical resistance of the thin film and the thermal barrier as expected. Also, the resistance of the device is higher than the non-treated bolometer, so we can easily choose 5 stable distinct bias conductivities of the YBCO thin film at the transition temperature. As YSZ needs a buffer layer for growing high quality YBCO on that, we made a $20 \mathrm{~nm}$ thick $\mathrm{Ce}_{0.9} \mathrm{La}_{0.1} \mathrm{O}_{2}$ (CLO) film as the buffer layer on the substrate before the deposition of the YBCO film by the same MOD method. The pattern of the bolometer is fabricated by standard photo lithography. The film is patterned to a meander line shape consisting of 15 lines, each line 50 $\mu \mathrm{m}$ wide and $1.5 \mathrm{~mm}$ long with spacing of $50 \mu \mathrm{m}$. Bolometer pattern is shown in Fig. 1 inset. A thin layer of gold film is also deposited on the four contact pads to make low resistance electrical contacts for the four-probe measurements.

We have used a TeraSense $95 \mathrm{GHz}$ source with canonical horn antenna and maximum output power of $75 \mathrm{~mW}$, for illuminating the bolometer. The source produces linearly polarized beam, and is modulated by a TTL signal for measurements versus modulation frequency. The measurements are carried out in a liquid nitrogen dewar with sapphire window, having high temperature stability in the range of $\mathrm{mK}$ and low noise considerations. Resistance and response of the fabricated bolometer are measured while the device is biased by low noise constant bias current in a standard four-probe configuration. Our measurement bandwidth is limited by the source modulation capabilities. Regarding that, the $95 \mathrm{GHz}$ source used here has $8 \mu$ s turn-on delay time; we have limited our measurements in this work to $3 \mathrm{kHz}$ starting from $0.2 \mathrm{~Hz}$ modulation frequencies.

The meander line pattern of the bolometer, makes it polarization dependent for relatively-long wavelength radiation used in this work. Indeed, we have measured the response of the device for two orthogonal directions of the source polarization relative to the direction of the meander lines of the TEB pattern.These two measurements have been done in the middle of superconducting transition where we have the maximum slope of the $R$ versus $T$ diagram. To investigate the effect of electrical conductivity of YBCO thin film on the detector response (e.g. the absorption efficiency of the meander line pattern, while it is parallel to the beam polarization) we have measured the response of the device thermally biased at 5 different points over the Superconductor-Normal transition region. By changing the thermal bias point of the device over the transition, the electrical conductivity of the YBCO film is altered, allowing measuring the radiation absorption efficiency of the device structure.

\section{RESULTS AND DISCUSSION}

According to the basic thermophysical model for the current biased TEB detectors [19], [20] the voltage responsivity is defined as:

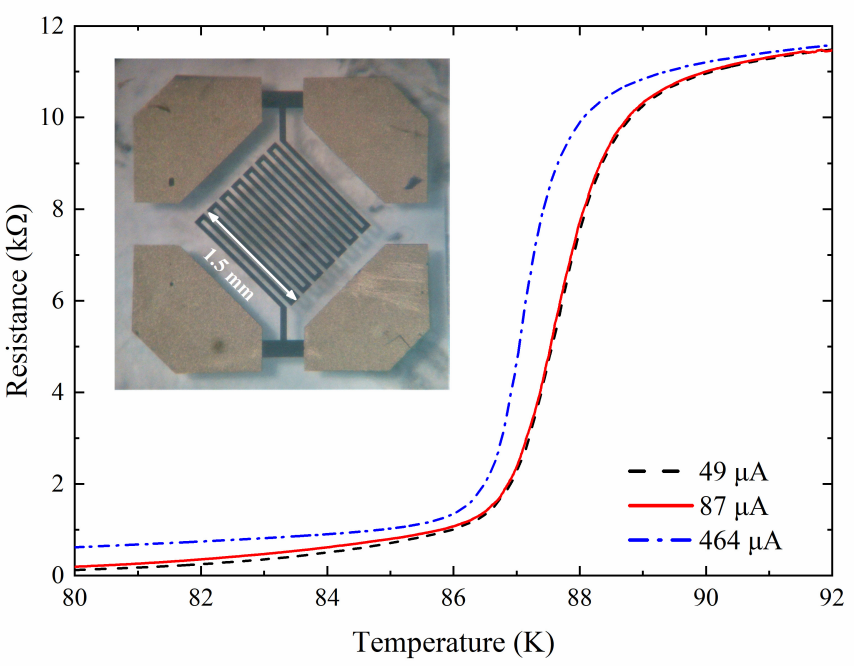

Fig. 1: Measured resistance versus biasing temperature $(R-T)$ of the fabricated Bolometer using four-probe technique for three different bias current. The inset shows the optical microscope image of the fabricated bolometer.

$$
r_{v}=\frac{\delta V}{\delta P}=\frac{\eta I_{b}}{G(1+i \omega \tau)} d R / d T
$$

where $\eta$ is the ratio of the absorbed power to the irradiated power, $I_{b}$ is the bias current, $G$ is the device total thermal conductance, $\tau=C / G$ is the time constant of the bolometer, and $C$ is the total heat capacity. Controlling thermal parameters of these detectors can significantly improve their responsivity [21]-[25]. Besides engineering the thermal parameters, the optical properties of the device play an important role in the absorption $\eta$ and consequently, the responsivity of the device for the target wavelength [26]-[28]. In this section we discuss these affecting parameters on the response of our device.

The results of the four-probe measurement of the device resistance indicates a relatively sharp transition for $\mathrm{YBCO}$ film with a transition width $\simeq 2 \mathrm{~K}$ as demonstrated in Fig. 1. Because of the intentionally applied roughness on the substrate, the $R-T$ measurement shows non-zero resistance phase as a tail in the $R-T$ curve at temperatures below 87 $\mathrm{K}$ which is not of concern in this paper. The resistance is measured for three different bias currents showing the effect of Joule heating of the device. The temperature rise in the YBCO film due to the Joule heating is governed by the equation $\Delta T=\frac{R I^{2}}{G(0)}$ with $R$ the resistance of the film, $I$ the bias current and $G(0)$ the dc thermal conductance of the system [29]. This effect can lead to an unreal sharper transition in the $R$ versus $T$ characterization at high bias currents. The shift in the measured $T_{c-\text { onset }}$ can be used to calculate $G(0)$ of the device. For our sample $G(0)$ is calculated to be $3.3 \mathrm{~mW} / \mathrm{K}$. As the results show, $I_{b}=464 \mu \mathrm{A}$ results in considerable Joule heating so that we have chosen $I_{b}=87 \mu \mathrm{A}$ for the response measurement that does not show a Joule heating effect but is high enough to enable low noise measurements. The response of the device radiated by $95 \mathrm{GHz}$ source with the meander lines of the pattern in parallel and perpendicular to the beam polarization is measured as shown in Fig. 2. We will mostly 


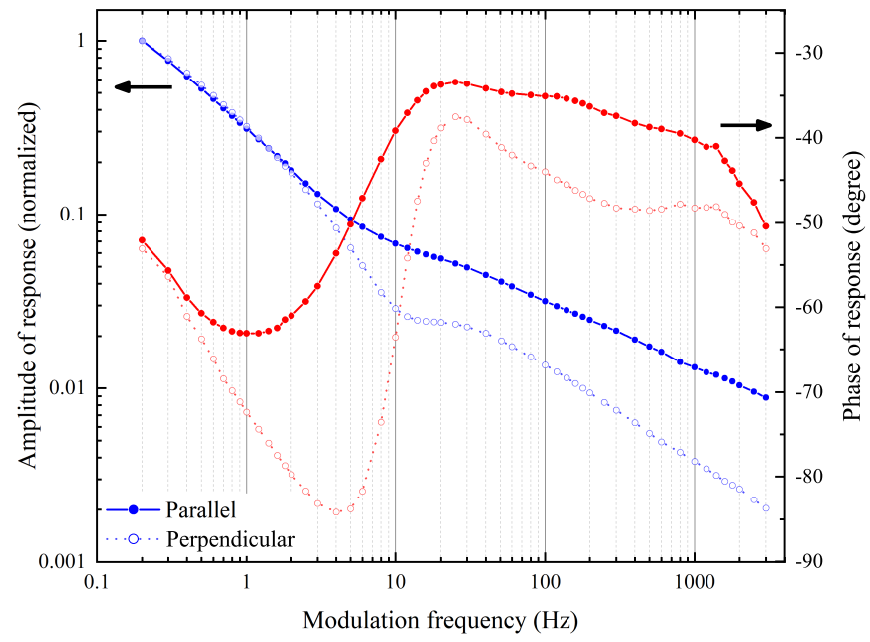

Fig. 2: Amplitude and phase of response voltage of the fabricated bolometer versus modulation frequency biased at $I_{b}=87 \mu \mathrm{A}$ and $T_{b}=88.3 \mathrm{~K}$. Solid and dotted lines belong to parallel and perpendicular cases respectively.

focus on the parallel case through this paper. The sample is biased at the middle of the transition at $88.3 \mathrm{~K}$ with dc current bias of $87 \mu \mathrm{A}$. The measured value of the noise-equivalentpower at $110 \mathrm{~Hz}$ modulation frequency has been obtained to be about $\mathrm{NEP} \approx 10^{-9} \mathrm{~W} / \sqrt{\mathrm{Hz}}$ and the corresponding responsivity to be $r_{v} \approx 13 \mathrm{~V} / \mathrm{W}$ which is comparable with the values of the reported devices [14], [30]-[32]. This kind of detectors has much potential for increase of the responsivity up to two orders of magnitude by increasing the biasing current and sharpening the superconducting transition width. Also, the pattern design can be further improved to achieve higher absorption.

We interpret that the measured response of the device to $95 \mathrm{GHz}$ is dominantly bolometric, and both the YSZ substrate and YBCO film play the role of absorber. The response versus modulation frequency curve (Fig. 2) has a knee point at $f_{k n e e}=5 \mathrm{~Hz}$ for the given bias and radiation parameters indicating that the thermal penetration length of the temperature variations due to the modulation frequency does not reach the substrate/cold-head interface of the dewar for higher frequencies and the response is mainly governed by the substrate and YBCO film and their interface properties and parameters in this range. For modulation frequencies below the knee frequency, the most dominant parameter that controls the response of the bolometer is the substrate-cold-head thermal conductance, $G_{s c}$. As expected, the knee frequency in the response versus modulation frequency of this YSZ substrate device is lower than that of the devices made on crystalline $\mathrm{LaAlO}_{3}$ and $\mathrm{SrTiO}_{3}$ and $\mathrm{MgO}$ which have higher thermal conductivity than that of YSZ [33]. In this region the bolometer can be modeled by a basic two-lumped-element R-C circuit with $R \simeq R_{s c}=\frac{1}{G_{s c}}$ and $C \simeq C_{\text {substrate }}$. The $f^{-1}$ behavior of basic R-C model can be seen in the response amplitude below the knee frequency.

Each meander line is $1.5 \mathrm{~mm}$ long that is half of the radiation wavelength. We speculate that the meander lines partly act as an antenna, interacting with the $95 \mathrm{GHz}$ radiation, and hence the amount of energy absorbed in the parallel direction results in higher response in this configuration compared to that of the perpendicular case. We have biased the device in the superconducting transition temperature range, so we have a superconducting gap approaching zero and many quasiparticles are present beside the Cooper pairs. Cooper pairs and quasi-particles can interact with the $95 \mathrm{GHz}$ radiation equal to a photon energy of $0.4 \mathrm{meV}$. They intake the photon energy and oscillate and hence reflect back some part of the absorbed energy, and convert the remaining part of the energy to phonons. When the direction of the meander lines is perpendicular to the beam polarization, the antenna effect is expected to decrease considerably and the measured response decreases accordingly.

In order to examine the antenna based absorption effect of the meander lines of the YBCO pattern, we have measured the phase and amplitude of the response versus modulation frequency of the device in five different bias temperatures in the superconducting transition region equivalent to five different conductivities of the YBCO film as shown in Fig. 3(a). The amplitude of the response at each bias point is divided by the $\mathrm{d} R / \mathrm{d} T$ of the film at that bias point to see the effect of the absorption of the device, shown in Fig. 3 (b). Experimental results show that there is an optimum electrical conductivity (or resistivity) for the film to get maximum coupling with the incident radiation. At lower temperatures where we approach a perfect conductor, the antenna is reflecting back most of the absorbed energy. At higher temperatures where the superconductivity disappears, the antenna does not absorb much of the energy due to the lower intake of the incident energy caused by relatively high resistivity. But, somewhere in the middle of the transition where we have a medium lossy conductor, the structure consisting of dielectric substrate and YBCO antenna absorbs maximum energy and reflects back the least. This maximum happens at $R=8700 \Omega$ equivalent to $\sigma=1.33 \times 10^{5} \mathrm{~S} / \mathrm{m}$ in our measurements as shown in Fig. 3(b). Similar effect in metamaterial based absorbers have been reported previously [34], [35]. In these reports, variation of the transmission spectrum of a meta-material structure with respect to the electrical conductivity of the silicon part of the meta-material unit cell is investigated. The results show that transmission minima will shift and the minimum value will change with changing the electrical conductivity of the silicon elements in the device.

Variations of the phase of the response versus modulation frequency is also shown in Fig. 3(b). At low modulation frequencies we see that the phase of the response is decreased by increasing temperature up to $89 \mathrm{~K}$ and the reason is already reported to be due to the effect of superconductivity transition on the substrate-cold-head conductance [36], [37]. At high modulation frequencies that the absorption of the superconducting film effect compared to that of the substrate becomes dominant, the phase is increased by increasing the bias temperature. In this regime, at low temperature part of the transition that Cooper pairs density is higher, we speculate to also have an intrinsic absorption mechanism due to the depairing of Cooper pairs that is fast resulting in lower phase 
a)
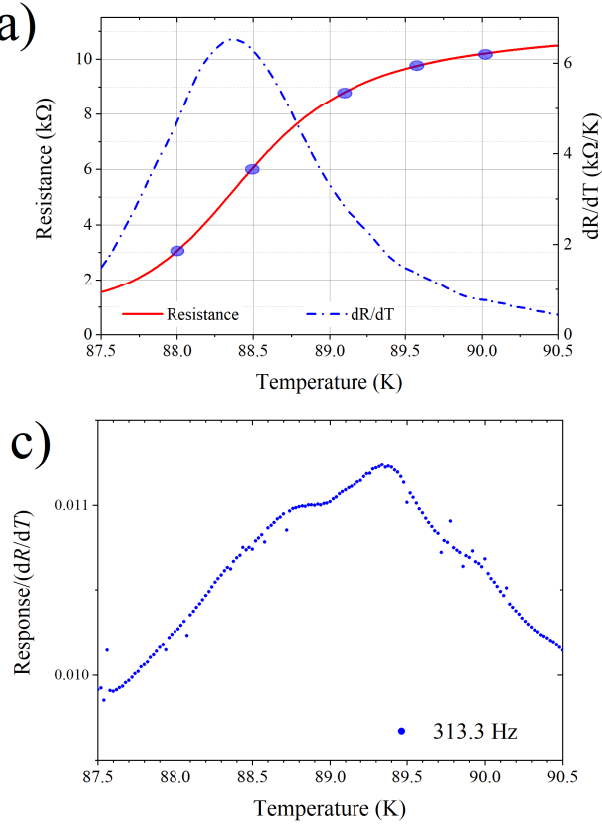

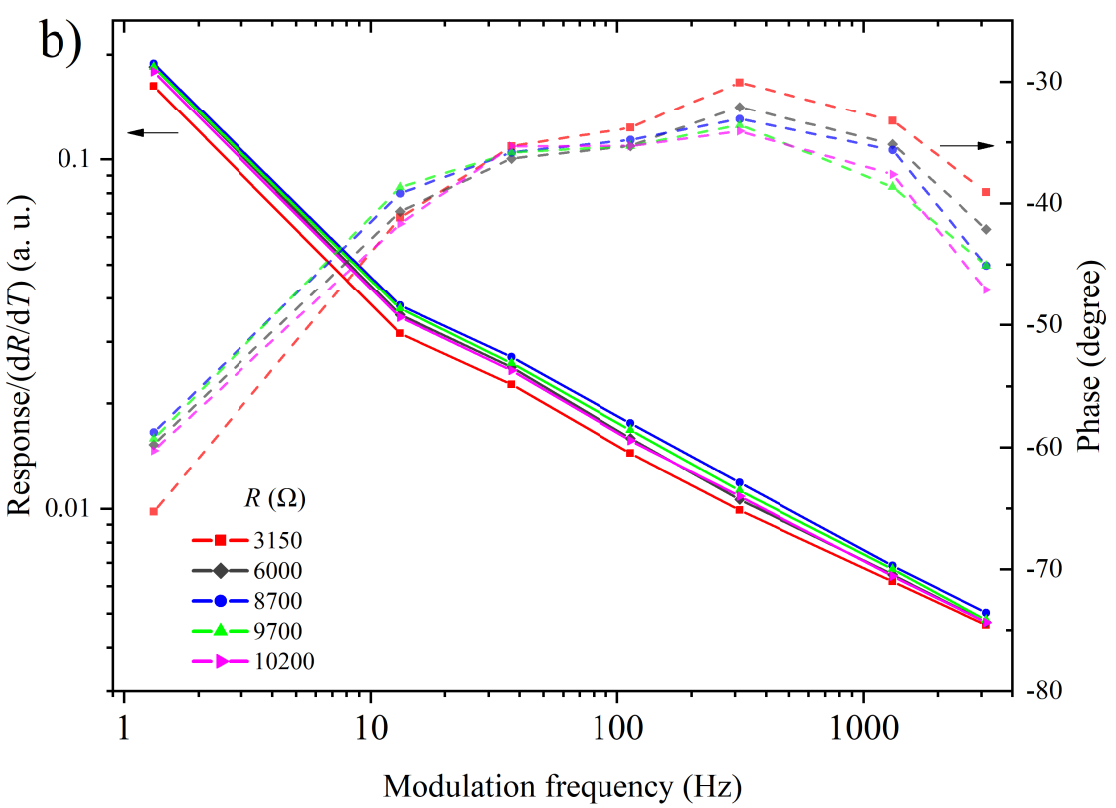

Fig. 3: a) Measured resistance and $\mathrm{d} R / \mathrm{d} T$ versus temperature at bias current $I_{b}=87 \mu \mathrm{A}$ when the detector is irradiated by the $95 \mathrm{GHz}$ source with $f=1.137 \mathrm{kHz}$ modulation frequency. Five temperature bias points used in plot (b) are shown in this figure. b) Amplitude of voltage response divided by $\mathrm{d} R / \mathrm{d} T$ (solid lines) and phase of voltage response (dashed line) measured versus modulation frequency for five distinct resistance of the YBCO film. c) Measured response of the device, divided to the corresponding $\mathrm{d} R / \mathrm{d} T$ versus temperature at modulation frequency $f=313.3 \mathrm{~Hz}$ and bias current $I_{b}=87 \mu \mathrm{A}$.

lag. Based on this approach, by increasing the bias temperature and having more normal electrons, our response becomes more bolometric due to the antenna dissipative effect resulting in higher phase lag. In another series of measurements we have swept the temperature to snap the superconductivity transition and we measured the response and resistance versus temperature simultaneously. The response is divided by the corresponding temperature $\mathrm{d} R / \mathrm{d} T$ to study the absorption versus temperature at each radiation modulation frequency. The resulting curve of this measurement for modulation frequency of $f_{m}=313.3 \mathrm{~Hz}$ is shown in Fig. 3(c). These results also reassert the existence of an optimum electrical conductivity for having maximum absorption of the radiated beam in our device.

The behavior of the absorption of our device is also further simulated in CST Microwave Studio softwareversion 2016.00, frequency domain solvers, to determine the absorption of this structure for various electrical conductivities of the YBCO film. Taking into consideration that our measurements and simulations are done at the superconducting transition temperature and the carriers are considered to be a mixture of Cooper pairs and quasi-particles [38], we have assumed a regular (lossy) conductor for the YBCO film with of course a correlated difference in the absorption due to the expected depairing/pairing mechanism in the material. Also, the required optical parameters of the YSZ substrate are extracted from ref. [39]. The simulations confirm experimental results showing that at $\sigma=1.33 \times 10^{5} \mathrm{~S} / \mathrm{m}$ we have the highest absorption and the lowest absorption at $\sigma=3.66 \times 10^{5} \mathrm{~S} / \mathrm{m}$ (Fig. 4 left axis) for the radiation frequency $f_{\text {rad }}=95 \mathrm{GHz}$. Right axis of Fig. 4 indicates the experimental counterpart of the absorption at each electrical conductivity for $f_{m}=313$ $\mathrm{Hz}$ inherited from Fig.3(b) for comparison. In this device the absorption maximum does not coincide with the maximum of the $\mathrm{d} R / \mathrm{d} T$ curve. Improving the absorption of the structure by geometrical optimization and shifting the absorption peak to the peak position of the $\mathrm{d} R / \mathrm{d} T$ curve can improve the maximum response of the device considerably, which is under further investigation. Ongoing research would generate detectors with higher responsivity and relatively simple structures with respect to the fabrication process and also with the capability of fabrication in large areas for the millimeter and sub-millimeter waves as a promising versatile $\mathrm{THz}$ and millimeter-wave detector with potentials for applications in medical and security imaging.

\section{SUMMARY AND CONCLUSION}

A monolithic meander line pattern YBCO TEB for $95 \mathrm{GHz}$ radiation frequency is designed, fabricated, and tested. The device, shows high response to the radiated power in the transition region of its YBCO film. Both amplitude and phase of the response of the detector versus modulation frequency is reported for bias temperature of $88.3 \mathrm{~K}$ being about the middle of superconducting transition. The response of the detector is measured to be higher when the meander lines are parallel to the direction of the beam polarization. We speculate that the YBCO film also acts as an antenna and absorbs the radiant energy more effectively when the meander lines are in parallel to the direction of the beam polarization. We have measured the response of the device in five different bias temperatures at the superconducting transition corresponding to five different electrical conductivities of the YBCO film, 


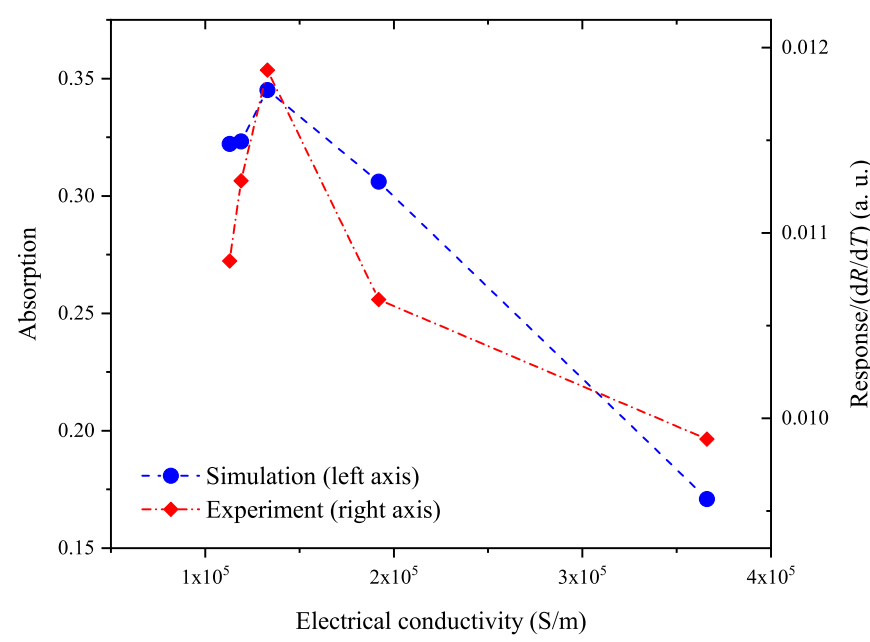

Fig. 4: Left axis (blue circles): simulation results for the absorption of the detector structure irradiated by $95 \mathrm{GHz}$ radiation. Right axis (red diamonds): Voltage response over $\mathrm{d} R / \mathrm{d} T$ measured at modulation frequency of $f=313$ $\mathrm{Hz}$.

while the meander lines are parallel to the beam polarization. The results of the normalized amplitude of the response to the $\mathrm{d} R / \mathrm{d} T$ of the film at the temperature bias points show that there is an optimum conductivity for having the highest absorption in the meander line patterned YBCO film. The analysis of the measured phase of the response also is in agreement with the discussed absorption mechanism. Finite element modeling of the absorption in the detector structure is also compatible with the experimental results showing that there is an optimal conductivity for having maximum absorption while this optimum conductivity does not coincide with the maximum of $\mathrm{d} R / \mathrm{d} T$ curve.

\section{ACKNOWLEDGMENT}

The authors would like to thank Mehdi Yaghoubi (Department of Electrical Engineering, Sharif University of Technology, Tehran, Iran) for his assistance for the response measurement setup.

\section{REFERENCES}

[1] J. Clarke, P. Richards, and N.-H. Yeh, "Composite superconducting transition edge bolometer," Applied Physics Letters, vol. 30, no. 12, pp. 664-666, 1977.

[2] M. Kenyon, P. Day, C. Bradford, J. Bock, and H. Leduc, "Backgroundlimited membrane-isolated TES bolometers for far-IR/submillimeter direct-detection spectroscopy," Nuclear Instruments and Methods in Physics Research Section A: Accelerators, Spectrometers, Detectors and Associated Equipment, vol. 559, no. 2, pp. 456-458, 2006.

[3] J. Brasunas and B. Lakew, "High- $\mathrm{T}_{\mathrm{c}}$ superconductor bolometer with record performance," Applied Physics Letters, vol. 64, no. 6, pp. 777-778, 1994.

[4] J. Zmuidzinas and P. L. Richards, "Superconducting detectors and mixers for millimeter and submillimeter astrophysics," Proceedings of the IEEE, vol. 92, no. 10, pp. 1597-1616, 2004

[5] B. S. Karasik, A. V. Sergeev, and D. E. Prober, "Nanobolometers for THz photon detection," IEEE Transactions on Terahertz Science and Technology, vol. 1, no. 1, pp. 97-111, 2011.

[6] J. Wei, D. Olaya, B. S. Karasik, S. V. Pereverzev, A. V. Sergeev, and M. E. Gershenson, "Ultrasensitive hot-electron nanobolometers for terahertz astrophysics," Nature Nanotechnology, vol. 3, no. 8, p. 496, 2008.
[7] Z. Schlesinger, R. Collins, D. Kaiser, and F. Holtzberg, "Superconducting energy gap and normal-state reflectivity of single crystal Y-Ba-CuO," Physical Review Letters, vol. 59, no. 17, p. 1958, 1987.

[8] P. Thoma, "Ultra-fast $\mathrm{YBa}_{2} \mathrm{Cu}_{3} \mathrm{O}_{7-\mathrm{x}}$ direct detectors for the $\mathrm{THz}$ frequency range," Ph.D. dissertation, 2013.

[9] H. Shibata, S. Kimura, S. Kashiwaya, S. Ueno, M. Koyanagi, N. Terada, E. Kawate, P. Fons, and Y. Tanaka, "Far-infrared optical conductivity of YBCO single crystal thin films from transmission and reflection spectra," Journal of Physics and Chemistry of Solids, vol. 62, no. 12, pp. 253-256, 2001.

[10] F. Gao, G. Carr, C. Porter, D. Tanner, S. Etemad, T. Venkatesan, A. Inam, B. Dutta, X. Wu, G. Williams et al., "Far-infrared transmittance and reflectance studies of oriented $\mathrm{YBa}_{2} \mathrm{Cu}_{3} \mathrm{O}_{7-\delta}$ thin films," Physical Review B, vol. 43, no. 13, p. 10383, 1991.

[11] S. Ali, L. D. Cooley, D. McCammon, K. L. Nelms, J. Peck, D. Prober, D. Swetz, P. T. Timbie, and D. van der Weide, "Planar antenna-coupled transition-edge hot electron microbolometer," IEEE Transactions on Applied Superconductivity, vol. 13, no. 2, pp. 184-187, 2003.

[12] S. Seliverstov, S. Maslennikov, S. Ryabchun, M. Finkel, T. Klapwijk, N. Kaurova, Y. Vachtomin, K. Smirnov, B. Voronov, and G. Goltsman, "Fast and sensitive terahertz direct detector based on superconducting antenna-coupled hot electron bolometer," IEEE Transactions on Applied Superconductivity, vol. 25, no. 3, pp. 1-4, 2014.

[13] A. Kuzmin, S. Doerner, S. Singer, I. Charaev, K. Ilin, S. Wuensch, and M. Siegel, "Terahertz transition-edge sensor with kinetic-inductance amplifier at $4.2 \mathrm{~K}$," IEEE Transactions on Terahertz Science and Technology, vol. 8, no. 6, pp. 622-629, 2018.

[14] A. Hammar, S. Cherednichenko, S. Bevilacqua, V. Drakinskiy, and J. Stake, "Terahertz direct detection in YBCO microbolometers," IEEE Transactions on Terahertz Science and Technology, vol. 1, no. 2, pp. 390-394, 2011.

[15] S. Seliverstov, S. Maslennikov, S. Ryabchun, M. Finkel, T. Klapwijk, N. Kaurova, Y. Vachtomin, K. Smirnov, B. Voronov, and G. Goltsman, "Fast and sensitive terahertz direct detector based on superconducting antenna-coupled hot electron bolometer," IEEE Transactions on Applied Superconductivity, vol. 25, no. 3, pp. 1-4, 2014.

[16] P. Thoma, J. Raasch, A. Scheuring, M. Hofherr, K. Il'in, S. Wünsch, A. Semenov, H.-W. Hübers, V. Judin, A.-S. Müller et al., "Highly responsive $\mathrm{Y}-\mathrm{Ba}-\mathrm{Cu}-\mathrm{O}$ thin film thz detectors with picosecond time resolution," IEEE Transactions on Applied Superconductivity, vol. 23, no. 3, pp. $2400206-2400206,2012$.

[17] L. Liu, H. Xu, R. R. Percy, D. L. Herald, A. W. Lichtenberger, J. L. Hesler, and R. M. Weikle, "Development of integrated terahertz broadband detectors utilizing superconducting hot-electron bolometers," IEEE Transactions on Applied Superconductivity, vol. 19, no. 3, pp. 282-286, 2009.

[18] R. Mohajeri, Y. A. Opata, A. C. Wulff, J.-C. Grivel, and M. Fardmanesh, "All metal organic deposited high $-\mathrm{T}_{\mathrm{c}}$ superconducting transition edge bolometer on yttria-stabilized zirconia substrate," Journal of Superconductivity and Novel Magnetism, vol. 30, no. 7, pp. 1981-1986, 2017.

[19] M. Fardmanesh, A. Rothwarf, and K. J. Scoles, "Low and midrange modulation frequency response for YBCO infrared detectors: Interface effects on the amplitude and phase," IEEE Transactions on Applied Superconductivity, vol. 5, no. 1, pp. 7-13, 1995.

[20] A. J. Kreisler and A. Gaugue, "Recent progress in high-temperature superconductor bolometric detectors: From the mid-infrared to the farinfrared (THz) range," Superconductor Science and Technology, vol. 13, no. 8, pp. 1235-1245, 2000.

[21] M. Hosseini, A. Kokabi, A. Moftakharzadeh, M. A. Vesaghi, and M. Fardmanesh, "Effect of substrate thickness on responsivity of freemembrane bolometric detectors," IEEE Sensors Journal, vol. 11, no. 12, pp. 3283-3287, 2011

[22] R. Mohajeri, R. Nazifi, A. C. Wulff, M. A. Vesaghi, J. C. Grivel, and M. Fardmanesh, "Investigation of $\mathrm{CeO}_{2}$ buffer layer effects on the voltage response of YBCO transition-edge bolometers," IEEE Transactions on Applied Superconductivity, vol. 26, no. 3, pp. 3-6, 2016.

[23] H. Neff, J. Laukemper, I. Khrebtov, A. Tkachenko, E. Steinbeiss, W. Michalke, M. Burnus, T. Heidenblut, G. Hefle, and B. Schwierzi, "Sensitive high- $\mathrm{T}_{\mathrm{c}}$ transition edge bolometer on a micromachined silicon membrane," Applied Physics Letters, vol. 66, no. 18, pp. 2421-2423, 1995.

[24] K. Li and J. E. Johnson, "Influence of substrate and biasing current on response of YBCO microbolometers," IEEE Transactions on Applied Superconductivity, vol. 3, no. 1, pp. 2111-2114, 1993.

[25] D. Fenner, Q. Li, W. Hamblen, M. Johansson, D. Hamblen, L. Lynds, and J. Budnick, "Optical and thermal performance advantages for silicon 
substrates in YBCO bolometer devices," IEEE Transactions on Applied Superconductivity, vol. 3, no. 1, pp. 2104-2106, 1993.

[26] A. Moftakharzadeh, A. Kokabi, A. Bozbey, T. Ghodselahi, M. A. Vesaghi, S. Khorasani, M. Banzet, J. Schubert, and M. Fardmanesh, "Investigation of bias current and modulation frequency dependences of detectivity of YBCO TES and the effects of coating of Cu-C composite absorber layer," IEEE Transactions on Applied Superconductivity, vol. 19, no. 4, pp. 3688-3693, 2009.

[27] S. Verghese, P. L. Richards, K. Char, and S. Sachtjen, "Fabrication of an infrared bolometer with a high $\mathrm{T}_{\mathrm{c}}$ superconducting thermometer," IEEE Transactions on Magnetics, vol. 27, no. 2, pp. 3077-3080, 1991.

[28] R. O'Brient, P. A. Ade, K. Arnold, G. Engargiola, W. Holzapfel, A. Lee, M. Myers, X. Meng, E. Quealy, P. Richards et al., "A multi-band dualpolarized antenna-coupled TES bolometer," Journal of Low Temperature Physics, vol. 151, no. 1-2, pp. 459-463, 2008.

[29] M. Fardmanesh, A. Rothwarf, and K. Scoles, " $\mathrm{YBa}_{2} \mathrm{Cu}_{3} \mathrm{O}_{7-\delta}$ infrared bolometers: Temperature-dependent responsivity and deviations from the dR/dT curve," Journal of Applied Physics, vol. 77, no. 9, pp. 4568-4575, 1995.

[30] D. Janik, D. May, H. Wolf, and R. Schneider, "High- $T_{C}$ transition-edge bolometer for detecting guided millimeter waves," IEEE transactions on applied superconductivity, vol. 3, no. 1, pp. 2148-2151, 1993.

[31] U. Oppenheim, M. Katz, G. Koren, E. Polturak, and M. Fishman, "High temperature superconducting bolometer," Physica C: Superconductivity, vol. 178 , no. $1-3$, pp. $26-28,1991$.

[32] S. Bevilacqua and S. Cherednichenko, "Low noise nanometer scale room-temperature $\mathrm{YBa}_{2} \mathrm{Cu}_{3} \mathrm{O}_{7-\mathrm{x}}$ bolometers for $\mathrm{THz}$ direct detection," IEEE Transactions on Terahertz Science and technology, vol. 4, no. 6, pp. 653-660, 2014.

[33] M. Fardmanesh, "Analytic thermal modeling for dc-to-midrange modulation frequency responses of thin-film high $-\mathrm{T}_{\mathrm{c}}$ superconductive edgetransition bolometers," Applied Optics, vol. 40, no. 7, pp. 1080-1088, 2001.

[34] H.-T. Chen, J. F. O'hara, A. K. Azad, A. J. Taylor, R. D. Averitt, D. B. Shrekenhamer, and W. J. Padilla, "Experimental demonstration of frequency-agile terahertz metamaterials," Nature Photonics, vol. 2, no. 5 , p. 295, 2008.

[35] J. Gu, R. Singh, X. Liu, X. Zhang, Y. Ma, S. Zhang, S. A. Maier, Z. Tian, A. K. Azad, H.-T. Chen et al., "Active control of electromagnetically induced transparency analogue in terahertz metamaterials," Nature Communications, vol. 3, no. 1, pp. 1-6, 2012.

[36] M. Fardmanesh and İ. N. Askerzade, "Temperature dependence of the phase of the response of YBCO edge-transition bolometers: Effects of superconductivity transition and thermal parameters," Superconductor Science and Technology, vol. 16, no. 1, p. 28, 2002.

[37] A. Bozbey, M. Fardmanesh, I. Askerzade, M. Banzet, and J. Schubert, "Effects of the superconductivity transition on the response of YBCO edge transition bolometers," Superconductor Science and Technology, vol. 16, no. 12, p. 1554, 2003.

[38] T. Kondo, Y. Hamaya, A. D. Palczewski, T. Takeuchi, J. Wen, Z. Xu, G. Gu, J. Schmalian, and A. Kaminski, "Disentangling Cooper-pair formation above the transition temperature from the pseudogap state in the cuprates," Nature Physics, vol. 7, no. 1, pp. 21-25, 2011.

[39] P. Smith and L. Davis, "Dielectric loss tangent of yttria stabilized zirconia at $5.6 \mathrm{GHz}$ and $77 \mathrm{~K}$," Electronics Letters, vol. 28, no. 4, pp. 424-425, 1992. 